\title{
An Approach to Temperature Dependence of the Magnetic Excitation in the Spin-Peierls Chain with Frustration
}

\author{
Xuefan JIANG*, G. Y. GUO ${ }^{1}$ and D. Y. XING ${ }^{2}$ \\ Department of Physics, Changshu Institute of Technology, Changshu 215500, China \\ ${ }^{1}$ Department of Physics, National Taiwan University, Taipei 106, Taiwan \\ ${ }^{2}$ National Laboratory of Solid State Microstructures, Nanjing University, Nanjing 210093, China
}

(Received October 29, 2003)

\begin{abstract}
In an adiabatic approximation we propose a theoretical method which combines the thermal coherent state with a quantum procedure to study temperature dependence of the magnetic excited gap in the spinPeierls chain with frustration based on the bosonized continuum Hamiltonian. In terms of the thermal coherent state the magnetic excitations at finite temperature can be viewed as the coherent excitations on an incoherent background constituted by the quasiparticles in thermal equilibrium. The formulations of the magnetic excited gap as a function of temperature are obtained analytically by solving a set of coupled equations. As an example, this method is applied to the spin-Peierls compound $\mathrm{CuGeO}_{3}$. It is shown that our theoretical results are well consistent with the inelastic neutron scattering experimental data for $\mathrm{CuGeO}_{3}$ in the normalized plane consisted of gap and temperature.
\end{abstract}

KEYWORDS: magnetic excitation, spin-Peierls chain, frustration, $\mathrm{CuGeO}_{3}$ DOI: $10.1143 / J P S J .73 .1561$

\section{Introduction}

There has been a great deal of interest in one-dimensional (1D) quantum spin systems since the discovery of a spinPeierls (SP) transition in the inorganic $\mathrm{CuGeO}_{3}$ compound. ${ }^{1)}$ This compound is believed to exhibit a SP transition at $T_{\mathrm{SP}}=14.2 \mathrm{~K}^{1,2)}$ Below this temperature the ground state is dimerized, and simultaneously a finite energy gap $\Delta$ opens between the nonmagnetic singlet ground state and the first excited triplet state and reaches the maximum value $\Delta \approx$ $2.15 \mathrm{meV}$ at $T=0 \mathrm{~K}^{-1}{ }^{1-5)}$ In the SP ordered phase the copper moments form singlet dimers along the chain with an energy gap to spin triplet excitations. Thermodynamical properties of pure and doped $\mathrm{CuGeO}_{3}$ have been examined in many experiments including specific heat ${ }^{6,7)}$ and magnetic susceptibility $^{8-10)}$ measurements.

To describe these properties, a $S=1 / 21 \mathrm{D}$ antiferromagnetic Heisenberg model of $\mathrm{CuGeO}_{3}$ with nearest neighbor (NN) and next-nearest-neighbor (NNN) interactions was proposed, ${ }^{11,12)}$ with the Hamiltonian in the form

$$
H=J \sum_{i=1}^{N}\left\{\left[1+(-1)^{i} \delta\right] \mathbf{S}_{i} \mathbf{S}_{i+1}+\alpha \mathbf{S}_{i} \mathbf{S}_{i+2}\right\},
$$

where $\mathbf{S}_{i}$ is the spin operator of site $i$ on chain, $N$ denotes the size of the chain, $J(>0)$ and $\alpha(>0)$ are the NN exchange integral and the ratio of the NNN exchange integral to the NN one, respectively. The parameter $\delta$ describes dimerization and $\alpha$ stands for the frustration. Below $T_{\mathrm{SP}}$, the value of temperature-dependent $\delta$ is nonzero. At zero temperature, Hamiltoian (1) was indicated that for $\delta>0$ or for $\alpha>\alpha_{c}$, with $\left.\alpha_{c}=0.2411,{ }^{13}\right)$ the singlet ground state is dimerized and a spin gap appears in the excitation spectrum while for $\alpha<\alpha_{c}$ and $\delta=0$ the system is gapless.

Since the discovery of $\mathrm{CuGeO}_{3}$ as the first inorganic SP compound there have been numerous studies of appropriate sets of microscopic couplings ${ }^{11,12,14)}$ within the $1 \mathrm{D}$ adiabatic approach. In order to estimate theoretically the $J$ and $\alpha$

*E-mail: jxfjxf@jlonline.com values, full diagonalization has been applied ${ }^{11,12,14)}$ to rings with $N \leq 18$. The finite-size data have been extrapolated to the thermodynamic limit and compared to the susceptibility measurement results above the SP transition point and from the singlet-triplet gap at zero temperature. Some estimates based on ab initio calculations of the electronic structure ${ }^{15,16)}$ have been also reported. Generally, from the direct calculations and the fitting to the experimental data, the estimates of the coupling spread over the interval $180 \mathrm{~K} \geq J \geq 135 \mathrm{~K}$. The value of $\alpha$ varies from 0.24 up to 0.45 . However, there is a dispersion of $\delta$ values at zero temperature previously reported in the literatures (between 0.01 and 0.12 ) $^{17,18 \text { ) }}$ depending on the model employed. A recently developed quantum transfer-matrix method has been used to estimate the temperature dependence of the dimerization parameter $\delta$ in the region below $T_{\mathrm{SP}}$ in $\mathrm{CuGeO}_{3}$. A set of parameters were derived as $J=166 \pm 2 \mathrm{~K}, \alpha=0.36 \pm 0.01$, and $\delta(T=$ $0)=0.022 \pm 0.002$. $^{19}$

Up to now, an enormous understanding of the low-energy physics of 1D SP systems has been reached, still it is a challenging task to investigative especially the low-temperature region of these systems, because for temperatures close to zero thermal fluctuations are strongly reduced and quantum fluctuations become dominant. At finite temperatures thermal fluctuations play an important role in the thermodynamic properties. However, the effect of the thermal fluctuation of the lattice distortion is not very clear for $T<T_{\mathrm{SP} .}{ }^{19)}$ In the SP state, as the temperature decreases from $T_{\mathrm{SP}}$, the dimerization develops and converges to the value of the dimerization of the ground state at $T=0$. Recently, both numerical techniques, i.e., the newly developed density matrix renormalization group (DMRG) ${ }^{20)}$ and quantum transfer-matrix (QTM) ${ }^{19)}$ have been used to study thermodynamical properties of $\mathrm{CuGeO}_{3}$ in the region of the finite temperature. However, most other numerical techniques such as the "traditional" Hamiltonian DMRG or exact diagonalization are restricted to either zero temperature or to rather large temperature. Theoretically, a simple molecular-field-type theory by mapping the low-energy 
solitonic excitations of dimerized spin chains on an effective Ising model was proposed to study the thermodynamical properties and the SP transition of $\mathrm{CuGeO}_{3} .{ }^{21)}$ In contrast, few analytical approaches have been presented to treat thermodynamical properties of the Hamiltonian (1) in region below $T_{\mathrm{SP}}$. It is therefore of interest to propose an effective analytical approach in studying the temperature dependence of magnetic excitation in the SP phase of this system.

Recently, a method based on both the concepts of coherent state and approach of the real time Green function was presented for handling the temperature field theory in 1D models. ${ }^{22}$ This method is essentially a two-fluid model. The coherent state corresponds to the superfluid component and the incoherent part corresponds to the normal fluid component. The incoherent thermal fluctuations increase with temperature and begin to destroy the long-range order until the latter totally vanishes at the critical temperature. Meanwhile, the thermal coherent state, as an alternative representation of the two-fluid model in relativistic field theory, was proposed by $\mathrm{Ni}$ et al. ${ }^{23)}$ It can be viewed as a mixed ensemble describing the coherent excitation on an incoherent background in thermal equilibrium, in which the accuracy of calculation is the same as that in the Green function approach to the lowest order. It does provide the advantage of simplifying the calculation and provide more direct insight into the physical problem. On the other hand, a quantum theory ${ }^{24)}$ was suggested to deal with the singlefrequency sine-Gordon (SG) model by applying a Bogoliubov transformation to the SG Hamiltonian; the condition of diagonalizing the quadratic terms leads to a self-consistent equation for the transformation parameter, in terms of which a special kind of ground state and the critical behavior of the model were described. The excitation spectrum with respect to the pairing ground state is characterized by an energy gap which is proportional to the transformation parameter. ${ }^{25)}$

In this paper we will start from the bosonized spin-Peierls Hamiltonian obtained by the Jordan-Wigner transformation for the 1D dimerized and frustrated Heisenberg model. We introduce the thermal coherent state to treat the effects of the thermal fluctuation. The magnetic excitation spectrum including the singlet-triplet excited gap as a function of temperature is derived analytically. The temperature dependence of the excitation energy gap is calculated for a set of microscopic couplings appropriate to the compound $\mathrm{CuGeO}_{3}$. Our results are compared with the experimental data in $\mathrm{CuGeO}_{3}$.

\section{Theory}

The theory is developed in three steps. First, by using a Jordan-Wigner transformation, the Hamiltonian (1) can be mapped onto a spinless fermion Hamiltonian, and the fermionic model is bosonized in the continuum limit. The resulting continuum Hamiltonian is given by ${ }^{26,27)}$

$$
H=\int \mathrm{d} x\left[A\left(\partial_{x} \phi\right)^{2}+C p^{2}-B \sin \phi+D \cos (2 \phi)\right],
$$

where $\phi$ and $p$ are a boson field operator and its canonical momentum operator, respectively. The coefficients $A, B, C$, and $D$ are related to $\delta$ and $\alpha$ by $A=a J(8 \pi)^{-1}(1+3 / \pi+$ $7 \alpha / \pi), \quad B=\delta J / a, \quad C=2 \pi a J(1-1 / \pi-\alpha / \pi), \quad$ and $\quad D=$ $J(2 a)^{-1}(1-3 \alpha)$ with $a$, a short distance cutoff or lattice spacing. If we make rescaling of the field operator and its conjugate momentum the Hamiltonian (2) can be transformed further into the standard form of the doublefrequency SG representation as, ${ }^{28)}$

$$
\begin{aligned}
H= & v \int \mathrm{d} x\left[\frac{1}{2}\left(\partial_{x} \phi\right)^{2}+\frac{1}{2} p^{2}\right. \\
& \left.-\frac{\lambda_{1}}{\eta^{2}} \cos (2 \eta \phi)-\frac{\lambda_{2}}{\eta^{2}} \cos (\eta \phi)\right],
\end{aligned}
$$

where $\lambda_{1}=-2 \pi a^{-2}(1-3 \alpha) /(\pi+3+7 \alpha), \lambda_{2}=4 \pi^{2} a^{-2} \delta /$ $(\pi+3+7 \alpha), \eta^{2}=4 \pi(\pi-1-3 \alpha)^{1 / 2} /(\pi+3+7 \alpha)^{1 / 2}$, and $v=a \pi^{-1} J(\pi+3+7 \alpha)^{1 / 2}(\pi-1-\alpha)^{1 / 2}$.

Since frustration and dimerization provide two principally different mechanisms for energy gap formation, we adjust, according to the ref. 29, coefficients of $\lambda_{1}$ and $\eta$ as $\lambda_{1}=$ $2 \pi a^{-2}\left(\alpha-\alpha_{c}\right) /(\pi+3+7 \alpha)$ and $\eta^{2}=2 \pi\left[1-\left(\alpha-\alpha_{c}\right)\right]^{1 / 2} /$ $\left[1+\left(\alpha-\alpha_{c}\right)\right]^{1 / 2}$. The following discussions are for the case of $\alpha \geq \alpha_{c}$.

Second, as mentioned above, we follow the definition of the thermal coherent state in ref. 23, by which the magnetic excitation at finite temperature can be regards as the coherent excitation of the quasiparticles on an incoherent background in thermal equilibrium for above boson system. The quasiparticles constitute an incoherent background on which we can construct a thermal coherent state as

$$
|f\rangle_{T}=\exp \int \mathrm{d} k\left[-\frac{1}{2} f_{k}^{*} f_{k}\left(1+n_{k}\right)+f_{k} b_{k}^{+}\right]|0\rangle_{T},
$$

here $b_{k}$ and $b_{k}^{+}$correspond to certain quasiparticle operators with momentum $k$ and $|0\rangle_{T}$ is given by the ensemble average ${ }_{T}\left\langle 0\left|b_{k}^{+} b_{k^{\prime}}\right| 0\right\rangle_{T}=\delta\left(k-k^{\prime}\right) n_{k}{ }^{30)} \quad$ with $\quad n_{k}=\left[\exp \left(\epsilon_{k} / k_{\mathrm{B}} T\right)-\right.$ $1]^{-1}$ standing for the Bose distribution function, where $\epsilon_{k}^{2}=$ $\mu^{2}+k^{2}$ with a mass $\mu$ which is arbitrary and temperature dependent. In order to make analytical calculation we take $\mu$ as the mass of the independent quasiparticle below. ${ }^{23)}$ So that as a good approximation, we have ${ }_{T}\left\langle 0\left|b_{k}^{+} b_{k^{\prime}}^{+}\right| 0\right\rangle_{T}=$ ${ }_{T}\left\langle 0\left|b_{k} b_{k^{\prime}}\right| 0\right\rangle_{T}=0$. The ensemble average of the observable $O$ may be calculated by $\langle O\rangle_{T}={ }_{T}\langle f|O| f\rangle_{T}$. Using the definition of $|0\rangle_{T}$ and the usual commutation relations of the annihilation and creation operators, the thermal coherent state average of the Hamiltonian (3) reads as

$$
\begin{aligned}
H_{T}= & v \int \mathrm{d} x\left[\frac{1}{2}\left(\partial_{x} \Phi\right)^{2}+\frac{1}{2} P^{2}\right. \\
& \left.-\frac{\lambda_{1 T}}{\eta^{2}} \cos (2 \eta \Phi)-\frac{\lambda_{2 T}}{\eta^{2}} \cos (\eta \Phi)\right],
\end{aligned}
$$

where $\Phi={ }_{T}\langle f|\phi| f\rangle_{T}, P={ }_{T}\langle f|p| f\rangle_{T}, \lambda_{1 T}=\lambda_{1} \mathrm{e}^{-4 \eta^{2} \Gamma}$, and $\lambda_{2 T}=\lambda_{2} \mathrm{e}^{-\eta^{2} \Gamma}$ with $\Gamma=(4 \pi)^{-1} \int_{0}^{\infty} \epsilon_{k}^{-1} n_{k} \mathrm{~d} k$. The dimensionless parameter $\Gamma$ plays the role of temperature-dependent mass renormalization. The integration of $\Gamma$ can be performed approximately with the following results ${ }^{31)}$

$$
\begin{array}{r}
\Gamma=\frac{1}{4 \pi}\left(\frac{\pi k_{\mathrm{B}} T}{2 \mu}\right)^{1 / 2} \mathrm{e}^{-\mu / k_{\mathrm{B}} T}\left[1-\frac{k_{\mathrm{B}} T}{8 \mu}+O\left(\frac{k_{\mathrm{B}}^{2} T^{2}}{\mu^{2}}\right)\right], \\
\text { for } \frac{\mu}{k_{\mathrm{B}} T} \gg 1,
\end{array}
$$

and

$$
\begin{array}{r}
\Gamma=\frac{1}{4 \pi}\left[\frac{\pi k_{\mathrm{B}} T}{2 \mu}+\frac{1}{2} \ln \frac{\mu}{4 \pi k_{\mathrm{B}} T}+\frac{1}{2} v+O\left(\frac{\mu^{2}}{k_{\mathrm{B}}^{2} T^{2}}\right)\right], \\
\text { for } \frac{\mu}{k_{\mathrm{B}} T} \ll 1,
\end{array}
$$


where $v=0.5772$ is the Euler constant. We note that $\lambda_{1 T}$ $\left(\lambda_{2 T}\right)$ is equal to $\lambda_{1}\left(\lambda_{2}\right)$ at the zero-temperature limit since $\Gamma=0$, so that eq. (5) is reduced to eq. (3) at $T=0 \mathrm{~K}$. As temperature increases the value of $\Gamma$ gradually increases and rapidly approaches infinite. A choice of $\mu=0$ would lead to divergence in eqs. (6) and (7) for which we would like to have $\lambda_{1 T}$ (or $\lambda_{2 T}$ ) $\rightarrow 0$ resulting in the mass of excited quasiparticle toward to zero, at which a transition occurs when temperature increases. We will set Boltzmann constant $k_{\mathrm{B}}=1$ below.

Third, contrary to the single-frequency SG model the theory of the double-frequency SG model is much less developed. For the case of zero temperature, a quantum theory was proposed ${ }^{24)}$ to deal with the single-frequency SG model based on the pairing quasiparticle states. The ground state can be realized with the help of a unitary transformation, and diagonalization procedure is essentially characterized by one parameter. The pairing ground state implies Bose condensation of the zero momentum mode, and the parameter is related to dynamical mass (or energy gap) generation. This method was expanded to the doublefrequency SG model describing dimerized and frustrated Heisenberg chain at zero temperature. ${ }^{28)}$ With it the properties of ground state and elementary excitation are given analytically. Our following investigation is based on this quantum theory. The field operator $\Phi(x)$ and its canonical momentum operator $P(x)$ are expanded in terms of the following scalar field ${ }^{24)}$

$$
\begin{aligned}
& \Phi(x)=\sum_{k}(2|k|)^{-1 / 2} \mathrm{e}^{-\zeta_{k}}\left(b_{k} \mathrm{e}^{\mathrm{i} k x}+b_{k}^{+} \mathrm{e}^{-\mathrm{i} k x}\right), \\
& P(x)=-\mathrm{i} \sum_{k}(|k| / 2)^{1 / 2} \mathrm{e}^{\zeta_{k}}\left(b_{k} \mathrm{e}^{\mathrm{i} k x}-b_{k}^{+} \mathrm{e}^{-\mathrm{i} k x}\right),
\end{aligned}
$$

which also obey the equal-time commutation relation: $\left[\Phi(x), P\left(x^{\prime}\right)\right]=\mathrm{i} \delta\left(x-x^{\prime}\right)$. The above definitions are different from the original expression for the field variable and its canonical momentum in terms of annihilation and creation operators, in which the infrared divergences induced by the massless scalar field have been considered with the help of the parameters $\zeta_{k}^{\prime}$ s. They are undetermined parameters related to the above field introduced. Normally ordering the two nonlinear interaction terms in eq. (5) gives the renormalization of the variable fugacity, ${ }^{32)}$ whose renormalization constant is

$$
\xi=\exp \left[-\eta^{2} / 4 \sum_{k} \mathrm{e}^{-2 \zeta_{k}} /|k|\right] .
$$

The diagonalization of the model Hamiltonian leads to get the following equation:

$$
\zeta_{k}=\frac{1}{4} \ln \left(1+4 \lambda_{1 T} \xi^{4} / k^{2}+\lambda_{2 T} \xi / k^{2}\right) .
$$

Substituting $\zeta_{k}$ into eq. (10), then $\xi$ is given self-consistently by

$$
\xi=\left\{\frac{4 \lambda_{1 T} \xi^{4}+\lambda_{2 T} \xi}{\left[1+\left(1+4 \lambda_{1 T} \xi^{4}+\lambda_{2 T} \xi\right)^{1 / 2}\right]^{2}}\right\}^{\eta^{2} / 8 \pi} .
$$

It should be pointed out that this quantum theory appropriately deals with the strong quantum fluctuations induced by the low energy excitations of quantum model, especially in the vicinity of the infrared divergency. ${ }^{24)}$ We can also evaluate the single-particle excitation spectrum as

$$
\omega(k)=\left(v^{2} k^{2}+\Delta^{2}\right)^{1 / 2},
$$

where $\Delta=v \mu$ standing for the energy gap of the singlettriplet excitation and $\mu$ is given by

$$
\mu=\left(4 \lambda_{1 T} \xi^{4}+\lambda_{2 T} \xi\right)^{1 / 2} \text {. }
$$

We have taken $a=1$. The appearance of a gap in the spectrum marks a dynamical mass generation from the freeboson model. When $\xi$ tends to zero, the gap (or mass) vanishes.

Now, we would like to point out that the calculation of the temperature dependence of the gap can not be obtained yet because the lattice dimerization as a function of the temperature, $\delta(T)$, is undetermined, which gives rise to difficulty for finding the temperature dependence of the gap. In general, in the adiabatic approximation, the dimerization should be determined by minimizing free energy of the coupled spin-lattice system, but, which is beyond the scope of our treatment. In present work, for simply, the properties of the magnetic excitation are main aim, although the elastic term have not been taken into account in the Hamiltonian (1). We note that usual scaling rules in Hamiltonian (1) without frustration have been developed by Cross and Fisher ${ }^{33)}$ to relate the temperature dependence of the spinPeierls energy gap to the dimerizing lattice distortion. This relates the energy gap and the superlattice intensity such that $\Delta \propto \delta^{\nu}$; in Cross-Fisher scaling $v=2 / 3$. However, Martin et $a l .{ }^{34)}$ found that, taking into account the accurate measurements by neutron-scattering for $\mathrm{CuGeO}_{3}$, this copper germanium system does not behave as a typical spin-Peierls system, which leads to a significant deviation from the Cross-Fisher scaling rule, suggesting the existence of frustration. Theoretically, at zero temperature, the DMRG results of Chitra et al. ${ }^{35)}$ gave a similar value $v=0.667 \pm$ 0.001 for the case of $\alpha=\alpha_{c}$ in terms of Hamiltonian (1). We have also studied analytically the same subject and found ${ }^{28)}$ that the behaviors of gap with dimerization obey the CrossFisher power law ${ }^{33)}$ only at $\alpha=\alpha_{c}$, but it is no longer valid for $\alpha>\alpha_{c}$, implying that the strong frustration in $\mathrm{CuGeO}_{3}$ gives rise to above deviation from the Cross-Fisher scaling rule. Furthermore, if we separate the definition of $\Delta$ into two parts, ${ }^{28)}$ i.e., $\Delta=\sqrt{\Delta_{\alpha}^{2}+\Delta_{\delta}^{2}}$, where $\Delta_{\alpha}=v \sqrt{4 \lambda_{1 T} \xi^{4}}$ and $\Delta_{\delta}=v \sqrt{\lambda_{2 T} \xi}$ which stand for the contributions of frustration and dimerization to the energy gap, respectively. Accordingly, the following power-law relation between $\Delta_{\delta}$ and $\delta(T)$ should be obeyed,

$$
\Delta_{\delta} / J=q \delta(T)^{2 / 3}
$$

where the coefficient $q$ can be determined by the value of parameters at zero temperature. Combining eq. (12) with eq. (15) we can get numerically the temperature dependences of the energy gap and dimerization.

\section{Results and Discussion}

Before discussing the temperature dependence of the energy gap, let us first focus our attention on variations of both parameters $(\xi$ and $\Gamma$ ) with temperature. Figure 1 shows the temperature dependences of $\xi$ and $\Gamma$ for selecting values of $\alpha=0.36$ and $\delta(0)=0.022$, in which solid and dashed 


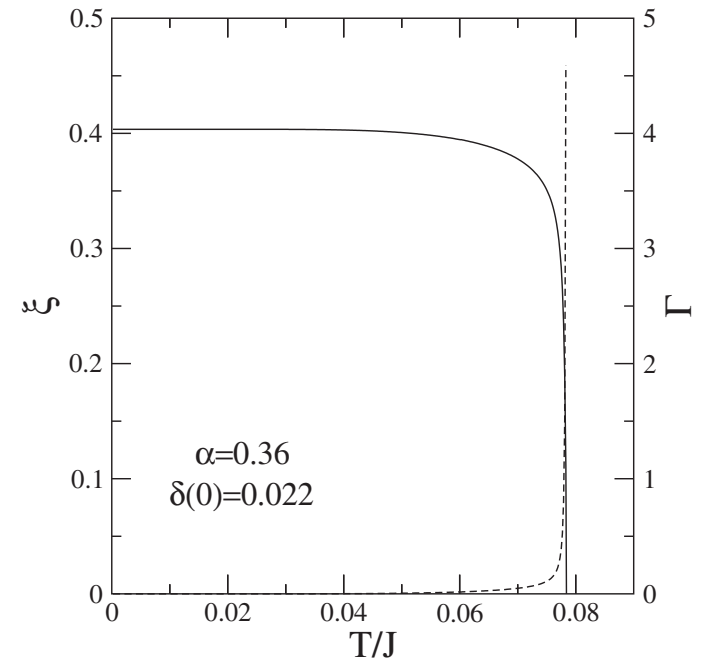

Fig. 1. The temperature dependences of two parameters of $\xi$ and $\Gamma$ for $\alpha=0.36$ and $\delta(0)=0.022$. Solid and dashed lines stand for $\xi$ and $\Gamma$, respectively.

lines stand for $\xi$ and $\Gamma$, respectively. It is found that as the temperature is increased, $\xi$ decreases gradually. At low temperature $\xi$ varies with temperature very slowly and $\Gamma$ is almost equal to zero. As the temperature is further increased, $\xi$ rapidly decreases to zero, while $\Gamma$ rapidly increases and approaches to infinite at a critical value of temperature. From the definition of the excited gap we can see that the both dimensionless parameters, $\xi$ and $\Gamma$, play the role of temperature-dependent gap renormalization, dominating strongly the temperature dependence of energy gap.

Figure 2 shows the temperature dependences of both the excited gap (solid line) and the dimerization (dashed line) for $\alpha=0.36$ and $\delta(0)=0.022$, in the units of exchange interaction. At zero temperature, it was found that the energy gap increases with increasing either frustration or dimerization parameter. ${ }^{28)}$ When the temperature is increased, the thermal fluctuations increase and gradually destroy the longrang order so that the energy gap decreases gradually. At low temperature the energy gap vary with temperature very

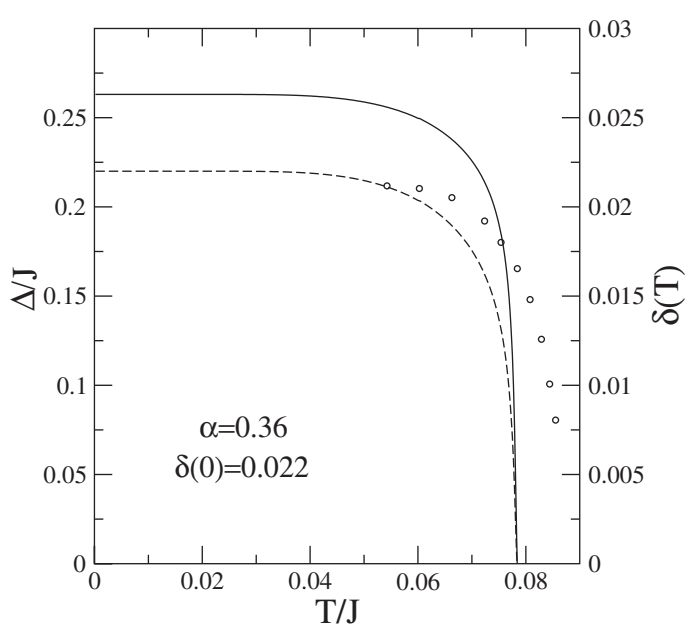

Fig. 2. Temperature dependence of both the energy gap $\Delta$ (solid line) and the dimerization parameter $\delta(T)$ (dashed line) for $\alpha=0.36$ and $\delta(0)=$ 0.022 in units of $J$. The circles stand for the temperature-dependent dimerization parameters from the $\mathrm{DMRG}^{20)}$ for $J=166 \mathrm{~K}$. slowly; while as the temperature is further increased, the energy gap rapidly decreases to zero, simultaneously the dimerization parameter becomes zero. There exists a SP transition temperature $T_{\mathrm{SP}}$, at which the dimerized system becomes undimerized due to strong thermal fluctuations. If we select $J=166 \mathrm{~K}$ for $\mathrm{CuGeO}_{3},{ }^{19)}$ it then follows that the singlet-triplet gap is equal to $\Delta=0.263 \mathrm{~J}=3.76 \mathrm{meV}$, somewhat greater than $\Delta^{\exp }=2.15 \mathrm{meV}$ as measured by inelastic neutron scattering experiment, ${ }^{3)}$ while the SP transition temperature is equal to $T_{\mathrm{SP}}=0.0784 \mathrm{~J}=$ $13.01 \mathrm{~K}$, which is consistent with the experimental value of $T_{\mathrm{SP}}^{\exp }=14.2 \mathrm{~K}$ for $\mathrm{CuGeO}_{3}$. In Fig. 2, circles stand for the temperature dependent dimerization parameters from the DMRG. ${ }^{20)}$ Overall, present calculated dimerization parameters coincide with those from the DMRG. ${ }^{20)}$ Here, we would like to point out that a strictly one-dimensional system shows theoretically no long-range order at finite temperature due to the solitonic excitations. The appropriate solitons in the SP state are domain walls in between two different dimer coverings, in which a dimer consists of a nearest-neighbor pair of spins in singlet state. ${ }^{21)}$ A single soliton in an otherwise (dimer-) order chain is in reality a complicated object, ${ }^{36)}$ which was spatially extended. ${ }^{37,38)}$ The finite-temperature transition to long-range order may be introduced by the interchain coupling. However, an indirect coupling of the chains via phonons is also conceivable in a mean-field approximation. ${ }^{39)}$ Comparing with experimental data, our results of the energy gap are slightly larger than measurement of experiment for $\mathrm{CuGeO}_{3}$. This is because there exists a weak interchain coupling in $\mathrm{CuGeO}_{3}{ }^{2)}$ As pointed out in ref. 36 , the interchain coupling may lead to an increase of soliton width, or a decrease of the energy gap. ${ }^{37)}$ In addition, if we consider simultaneously the effect of interchain coupling, the above set of microscope parameters ought to be renormalized by the interchain coupling. ${ }^{11)}$ On the other hand, we have also neglected the renormalization of the parameters in transforming the lattice model eq. (1) into the continuum model eq. (2). ${ }^{40)}$ The renormalized values of the parameters in the continuum models can be found by solving the appropriate renormalization ground equations. ${ }^{41)}$ A detailed discussion on these problems is beyond the scope of the present paper. For these reasons, we will therefore consider direct comparison between our theoretical results and experimental data in the normalized plane consisted of the energy gap and temperature below.

In Fig. 3 we plot the temperature-dependent energy gap in normalized space, in which squares stand for the experimental data from the inelastic neutron scattering experiment by Regnault et $a l .{ }^{3)}$ The agreement between experimental and theoretical data is good. This behavior is very much like the BCS superconducting theory, implying the similarity between the Peierls and the BCS systems. In fact, the ground state wave function in terms of the above self-consistent theory can be constructed as $|G\rangle=\exp \sum_{k}\left(\zeta_{k} / 2\right)\left(b_{k} b_{-k}-\right.$ $\left.b_{k}^{+} b_{-k}^{+}\right)|\mathrm{vac}\rangle .^{24,25)}$ We can see that the ground state is a paring boson state characterized by an excited spectrum with a gap, which is analogous to the BCS superconducting state. According to the definition of $\zeta_{k}$ we can also see that the pairing state will return to a no excited particle state when the parameter $\xi$ tends to zero, which corresponds to the destruction of the pairing quasiparticle state. At this point 


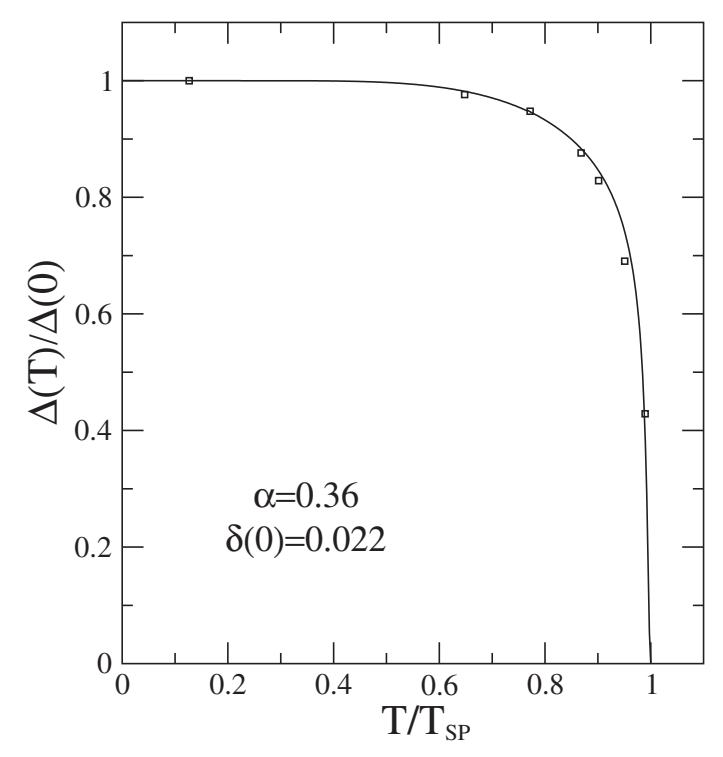

Fig. 3. The normalized gap $\Delta(T) / \Delta(0)$ vs the normalized temperature $T / T_{\mathrm{SP}}$. Squares are experimental data in $\mathrm{CuGeO}_{3}$ by inelastic neutron scattering experiment from ref. 3 .

system undergoes a transition from a gap one to a gapless one. When temperature increases the parameter $\xi$ drops rapidly near a critical temperature, simultaneously the parameter $\Gamma$ increases rapidly and approaches to infinite, leading to the energy gap of system disappearing at the critical temperature, see Fig. 1. In terms of the relation of $\Delta_{\delta} \propto \delta^{2 / 3}$, the lattice order parameter, i.e., $\delta(T)$, becomes continuously zero at transition temperature $T_{\mathrm{SP}}$, indicating the behavior of second-order transition. Experimentally, in the vicinity of $T_{\mathrm{SP}}$, it was found ${ }^{2,3)}$ that the temperature dependence of the energy gap in $\mathrm{CuGeO}_{3}$ does not agree with the BCS equation $\Delta(T) / \Delta(0) \propto\left(1-T / T_{\mathrm{C}}\right)^{1 / 2}$ as $T \longrightarrow T_{\mathrm{C}}$ (and $T<T_{\mathrm{C}}$ ) based on the mean-field theories and a much smaller critical exponent exists, although the equation $2 \Delta(0) \approx 3.50 k_{\mathrm{B}} T_{\mathrm{SP}}$ is very close to the BCS formula $2 \Delta(0)=3.53 k_{\mathrm{B}} T_{\mathrm{C}}$. These phenomena are caused by a $1 \mathrm{D}$ quantum spin fluctuation in $\mathrm{CuGeO}_{3}$. On the other hand, the neutron scattering measurements for $\mathrm{CuGeO}_{3}$ indicate near $T_{\mathrm{SP}}$ a good fit of the energy gap data to $\Delta(T) \propto$ $\left(T_{\mathrm{SP}}-T\right)^{0.66 a}$ with $a \approx 1 / 6 .^{34)}$ This result indicts that $\mathrm{CuGeO}_{3}$ is not a typical spin-Peierls system in which the Cross-Fisher scaling rule holds. From Fig. 3 we have also analyzed our calculated results in the vicinity of $T_{\mathrm{SP}}$ starting from a conventional power-low behavior given by the relation $\Delta(T) / \Delta(0) \propto\left(1-T / T_{\mathrm{SP}}\right)^{\beta}$. The best fit to our results is achieved with parameter $\beta \approx 0.17$, in good agreement with experimental measurements for $\mathrm{CuGeO}_{3}{ }^{2,3}$ ) and close to the fit results obtained by neutron scattering measurements. ${ }^{34,42)}$ This good agreement between theory and experiment lends further support to present approach to the temperature dependence of the magnetic excitation of the spin-Peierls chain with frustration. ${ }^{43}$

\section{Summary}

Based on the bosonized Hamiltonian we have proposed an analytical method which combines the thermal coherent state with a quantum procedure to calculate temperature dependence of the energy gap of the triplet excited state in the spin-Peierls chain with frustration. We have numerically studied the temperature dependence of the magnetic excitation in the spin-Peierls compound $\mathrm{CuGeO}_{3}$ in terms of dimerized and frustrated antiferromagnetic Heisenberg chain under the adiabatic approximation. With the help of a definition of the thermal coherent state, the magnetic excitation at finite temperature can be regarded as a coherent excitation of the quasiparticles on an incoherent background in thermal equilibrium. Qualitatively, our theoretical results are in good agreement with those from the inelastic neutron scattering experiments of $\mathrm{CuGeO}_{3}$. The behavior of the energy gap which depends on the temperature for $\mathrm{CuGeO}_{3}$ is similar to BCS superconducting theory.

As a conclusion, in present theory, we would also like to point out that, from a point of view in quantum field theory, some information about the nature of gap (or mass) and its relevance to the critical temperature can be obtained because of the introduction of the thermal coherent state. At a certain temperature, the mass of elementary excitations receives two contributions added together. One is coherence resulting from spontaneously broken symmetry and may be weakly dependent on the temperature. The other is incoherent and strongly dependent on the temperature. Then a critical temperature may exist, above which the symmetry broken system restores to the original symmetric state accompanied by the disappearance of excited energy gap in the boson system. Overall, our theory gives a kind of description of the magnetic excitation in the spin-Peierls chain with frustration such as the compound $\mathrm{CuGeO}_{3}$ at finite temperature.

\section{Acknowledgments}

X.F.J. was supported in part by the Natural Science Foundation of Jiangsu Educational Department of China under Grant No. 03KJB140001. G.Y.G. was supported in part by the National Science Council of Taiwan under Grant No. NSC91-2816-M-002-0009-6. D.Y.X. was supported in part by the Natural Science Foundation of China.

1) M. Hase, I. Terasaki and K. Uchinokura: Phys. Rev. Lett. 70 (1993) 3651 .

2) M. Nishi, O. Fujita and J. Akimitsu: Phys. Rev. B 50 (1994) 6508.

3) L. P. Regnault, M. Ain, B. Hennion, G. Dahlenne and A. Revcolevschi: Phys. Rev. B 53 (1996) 5579.

4) M. Arai, M. Fujita, M. Motokawa, J. Akimitsu and S. M. Bennington: Phys. Rev. Lett. 77 (1996) 3649.

5) M. Aïn, J. E. Lorenzo, L. P. Regnault, G. Dhalenne, A. Revcolevschi, B. Hennion and Th. Jolicoeur: Phys. Rev. Lett. 78 (1997) 1560.

6) J. C. Lasjaunias, P. Monceau, G. Remenyi, S. Sahling, G. Dahlenne and A. Revcolevschi: Solid State Commun. 101 (1997) 677.

7) H. Kuroe, J. Sasaki, T. Sekine, N. Koide, Y. Sasago, K. Uchinokura and M. Hase: Phys. Rev. B 55 (1997) 409.

8) J. P. Pouget, L. P. Regnault, M. Aïn, B. Hennion, J. P. Renard, P. Veillet, G. Dahlenne and A. Revcolevschi: Phys. Rev. Lett. 72 (1994) 4037.

9) B. Grenier, J. P. Renard, P. Veillet, C. Paulsen, R. Calemczuk, G. Dahlenne and A. Revcolevschi: Phys. Rev. B 57 (1998) 3444.

10) P. E. Anderson, J. Z. Liu and R. N. Shelton: Phys. Rev. B 56 (1997) 11014.

11) J. Riera and A. Dobry: Phys. Rev. B 51 (1995) 16098.

12) G. Castilla, S. Chakravarty and V. J. Emery: Phys. Rev. Lett. 75 (1995) 1823.

13) K. Okamoto and K. Nomura: Phys. Lett. A 169 (1993) 433.

14) K. Fabricius, A. Klümper, U. Löw, B. Büchner, T. Lorenz, G. Dhalenne and A. Revcolevschi: Phys. Rev. B 57 (1998) 1102.

15) L. F. Mattheiss: Phys. Rev. B 49 (1994) 14050. 
16) S. Zagoulaev and I. I. Tupitsyn: Phys. Rev. B 55 (1997) 13528.

17) R. Werner, C. Gros and M. Braden: Phys. Rev. B 59 (1999) 14356.

18) B. Büchner, H. Fehske, A. P. Kampf and G. Wellein: Physica B 259 (1999) 956.

19) G. Kamieniarz, M. Bielinski and J. P. Renard: Phys. Rev. B 60 (1999) 14521.

20) A. Klümper, R. Raupach and F. Schönfeld: Phys. Rev. B 59 (1999) 3612.

21) R. Werner and C. Gros: Phys. Rev. B 57 (1998) 2897.

22) R. K. Su, P. Z. Bi and G. J. Ni: J. Phys. A 16 (1983) 2445.

23) G. J. Ni, J. J. Xu and W. Chen: J. Phys. A 18 (1985) 149.

24) G. M. Zhang, H. Chen and X. Wu: Phys. Rev. B 43 (1991) 13566.

25) Y. M. Zhang, M. L. Zhou and B. W. Xu: Phys. Rev. B 47 (1993) 898.

26) S. Inagaki and H. Fukuyama: J. Phys. Soc. Jpn. 52 (1983) 3620.

27) K. Kuboki and H. Fukuyama: J. Phys. Soc. Jpn. 56 (1987) 3126

28) X. F. Jiang, H. Chen, D. Y. Xing and J. Dong: J. Phys.: Condens. Matter 13 (2001) 6519.

29) G. Bouzerar, A. P. Kampf and G. I. Japaridze: Phys. Rev. B 58 (1998) 3117.

30) J. F. Donoghue and B. R. Holstein: Phys. Rev. D 28 (1983) 340.

31) L. Dolan and R. Jackiw: Phys. Rev. D 9 (1974) 3320.

32) S. Coleman: Phys. Rev. D 11 (1975) 2088.
33) M. C. Cross and D. S. Fisher: Phys. Rev. B 19 (1979) 402.

34) M. C. Martin, G. Shirane, Y. Fujii, M. Nishi, O. Fujita, J. Akimitsu, M. Hase and K. Uchinokura: Phys. Rev. B 53 (1996) R14713.

35) R. Chitra, S. Pati, H. R. Krishnamurthy, D. Sen and S. Ramasesha: Phys. Rev. B 52 (1995) 6581.

36) B. S. Shastry and B. Sutherland: Phys. Rev. Lett. 47 (1981) 964.

37) J. Zang, S. Chakravarty and A. P. Bishop: Phys. Rev. B 55 (1997) R14705.

38) A. Dorby and J. A. Riera: Phys. Rev. B 56 (1997) R2912.

39) E. Pytte: Phys. Rev. B 10 (1974) 2039; ibid. 10 (1974) 4637.

40) H. J. Schulz: Phys. Rev. B 34 (1986) 6372.

41) J. L. Black and V. J. Emery: Phys. Rev. B 23 (1981) 429.

42) J. G. Lussier, S. M. Coad, D. F. McMorrow and D. McK Paul: J. Phys.: Condens. Matter 8 (1996) L59.

43) Note that the mean-field theory ${ }^{44)}$ of $\delta=\Delta / r J$ with $r=1.637$ results in a larger discrepancy in the value of $\Delta$ at zero temperature in comparison to those coming from experimental measurements of $\mathrm{CuGeO}_{3}$.

44) J. W. Bray, H. R. Hart, L. V. Interrante, I. S. Jacobs, J. S. Kasper, G. D. Watkins, S. H. Wee and J. C. Bonner: Phys. Rev. Lett. 35 (1975) 744 . 